\title{
Natural killer cells protect mice from DSS-induced colitis by regulating neutrophil function via the NKG2A receptor
}

\author{
LJ Hall ${ }^{1,2}$, CT Murphy ${ }^{1}$, A Quinlan ${ }^{1}$, G Hurley ${ }^{1}$, F Shanahan ${ }^{1}, K_{\text {Nally }}^{1,3}$ and S Melgar ${ }^{1,3}$
}

Natural killer (NK) cells are traditionally considered in the context of tumor surveillance and infection defense but their role in chronic inflammatory disorders such as inflammatory bowel disease is less clear. Here, we investigated the role of NK cells in dextran sodium sulfate (DSS)-induced colitis in mice. Depletion of NK cells impairs the survival of mice with colitis and is linked with dramatic increases in colonic damage, leukocyte infiltration, and pro-inflammatory profiles. Mice depleted of NK cells had increased numbers of neutrophils in colons and mesenteric lymph nodes, compared with control mice, in addition to acquiring a hyper-activation status. In vitro and in vivo studies demonstrate that NK cells downregulate pro-inflammatory functions of activated neutrophils, including reactive oxygen species and cytokine production, by direct cell-to-cell contact involving the NK cell-inhibitory receptor NKG2A. Our results indicate an immunoregulatory mechanism of action of NK cells attenuating DSS-induced colitis neutrophil-mediated inflammation and tissue injury via NKG2A-dependent mechanisms.

\section{INTRODUCTION}

Natural Killer (NK) cells are a subset of innate lymphocytes that contribute to host resistance to viruses, bacteria and certain parasites and provide immune surveillance against the development of tumors. The lytic activity of NK cells is controlled by complex interactions of inhibitory and activating receptors with specialised signaling machinery. In the mouse, NK cellactivating receptors include NKG2D, 2B4, and NKp46, which recognize target cell expressed ligands and trigger NK cellmediated cytotoxicity. Inhibitory receptors, such as Ly49A and NKG2A, recognize MHC (major histocompatibility complex) class I molecules and enable blockade of NK cell activation. ${ }^{1}$ Alongside their cytotoxic function, NK cells also produce a wide range of cytokines and immunomodulators, including tumor necrosis factor- $\alpha$ and interferon (IFN) $-\gamma^{2}$. During inflammation, cross-talk with accessory cells, such as dendritic cells or macrophages, and more recently neutrophils, is required for full activation of NK cells. This activation can work both ways with cytokine release via NK cells modulating both dendritic cell and macrophage function, therefore actively contributing to the generation of the subsequent immune response. ${ }^{2-4}$
Appreciation for the participation of NK cells in a number of chronic inflammatory diseases has grown recently. A protective role for NK cells has been reported in animal models of diabetes, ${ }^{5}$ experimental autoimmune encephalomyelitis/multiple sclerosis, ${ }^{6}$ and systemic lupus erythematosus. ${ }^{7}$ By contrast, NK cells have been shown to promote inflammation in models of arthritis ${ }^{8}$ and chronic obstructive pulmonary disease. ${ }^{9}$ Thus, the role of NK cells in inflammatory disease appears to vary and may be context-dependent.

Ulcerative colitis (UC) is a chronic relapsing and remitting inflammatory condition of the large bowel with an approximate prevalence of 1:1,000 in developed countries and increases with socioeconomic development. Although the pathogenesis of UC is still incompletely understood, previous work indicates a complex interplay of genetic and environmental factors with a dysregulated immune response. ${ }^{10}$ Increases in acute disease activity in UC is linked to a dense influx of neutrophils into the mucosa and intestinal lumen, resulting in the formation of socalled crypt abscesses. ${ }^{11}$ In addition, circulating activated neutrophils, a major source of inflammatory cytokines, are elevated in active UC, and neutrophil reactive oxygen species

\footnotetext{
${ }^{1}$ Alimentary Pharmabiotic Centre, University College Cork, National University of Ireland, Cork, Ireland and ${ }^{2}$ Norwich Medical School, University of East Anglia, Norwich Research Park, Norwich, UK. Correspondence: LJ Hall (Lindsay.Hall@uea.ac.uk); S Melgar (s.melgar@ucc.ie)

${ }^{3}$ These authors share joint senior authorship of this work.
} 
(ROS) production is associated with acute pathology in UC as well as in a number of chronic inflammatory conditions. ${ }^{12}$

Conventional NK cells appear to have a role during colitis, but the importance of their relative contribution is unclear. In the mouse transfer model of chronic colitis, interleukin (IL)$10^{-1-} \mathrm{CD} 4^{+} \mathrm{T}$ cells expanded more rapidly and caused more severe disease in $\mathrm{NK}$ cell-depleted $\mathrm{RAG}^{-/-}$recipients. ${ }^{13}$ Recently, a study in T-cell-reconstituted $\mathrm{RAG}^{-/-}$-recipient mice, suggests that NK cells can supress colitis severity through targeting of colitogenic $\mathrm{T}$ effector memory cells and possibly early T-cell developmental stages. ${ }^{14}$ Whereas, in athymic nu/nu (nude) mice, NK cells did not appear to have a role in suppressing development of chronic colitis. ${ }^{15}$ Previous studies in UC patients have provided conflicting results with regards to NK cell numbers. ${ }^{16,17}$ However, blood-derived NK cells from UC patients appear to have functional defects, including impairment of cytotoxicity. ${ }^{18}$

To determine the contribution of more conventional NK cells in colitis, we studied the response of two NK cell-deficient (NKD) mice to dextran sodium sulfate (DSS)-induced experimental colitis. Hallmarks of this model include epithelial disruption, resulting in overproduction of inflammatory cytokines, release of reactive oxygen metabolites, and infiltration of granulocytes into the colon, resulting in colonic inflammation and tissue damage. These hallmarks resemble a number of features of acute mucosal injury in human UC. ${ }^{19,20}$ Our results demonstrate that NK cells are rapidly recruited to the inflamed colon and are protective during DSS-induced colitis. NK cells mediate this protective effect by downregulating neutrophil function via the inhibitory receptor NKG2A. These findings suggest that NK cells can also use their array of activation and inhibitory receptors to directly modulate other immune populations, which may serve to selectively downregulate potentially damaging inflammatory responses.

\section{RESULTS}

\section{NKD mice rapidly succumb to DSS-induced colitis}

To determine whether NK cells could traffic directly to the inflamed colon, NK cells were isolated from DSS-treated transgenic $\beta$-actin luciferase ${ }^{+}$donor animals and intravenously injected into DSS-treated or healthy recipients. Transferred NK cells accumulated within mesenteric lymph nodes (MLN) and colons of DSS-treated mice when compared with healthy recipients (Figure 1a and Supplementary Figure S1). Moreover, flow cytometry and immunofluorescence revealed significant $(P<0.05)$ increases in NK cells within the MLN and colons as early as day 1 post-DSS administration, with concurrent expression of a wide range of cytokine and cytotoxic factors during DSS-induced colitis (Supplementary Figure S2).

To demonstrate a role for NK cells during DSS-induced colitis, polyclonal anti-asialo GM1 (anti-AGM1) antibody was used to actively deplete the NK cell population from murine tissues in vivo. ${ }^{4}$ In addition, we used mice with a selective NK cell deficiency (NKD mice). ${ }^{21}$ Unexpectedly, both
anti-AGM1-treated (NK cell-depleted) and NKD mice rapidly succumbed to DSS-mediated colitis, whereas immunoglobulin G (IgG) control or wild-type (NK cell ${ }^{+}$) mice did not (Figure 1b). Concurrently, both body weight loss and disease activity index of these NKD mice were significantly greater $(P<0.001)$ than that of control DSS mice (Figure 1c and Supplementary Figure S3). On day 7, both anti-AGM1-treated and NKD DSS mice had significantly heavier $(P<0.01)$ colons and a significantly greater $(P<0.05)$ spleen mass when compared with $\mathrm{NK}$ cell ${ }^{+}$control DSS mice (Figure 1d and Supplementary Figure S3). Both NKdepleted and NKD mice produced significantly elevated $(P<0.05)$ levels of colonic IL-17A, IL-6, mKC (mouse keratinocyte-derived chemokine), and IL-1 $\beta$ but reduced $(P<0.05)$ IFN- $\gamma$, IL-10, transforming growth factor- $\beta$ and gzmB (granzyme B) compared with control DSS animals (Figure 1e). We did not detect any significant differences between the DSS groups for IL-22, suggesting that IL-22 production from the resident ROR $\gamma$ t subset population and/or the presence of activated T cells cannot explain why NKD mice have increased bowel pathology in our model ${ }^{22,23}$ (Figure 1e). Histological examination and scoring of colon sections revealed significantly $(P<0.05)$ greater crypt and epithelial cell loss, thickening of the intestinal wall with considerable mixed cell infiltration in both NK-depleted and NKD mice compared with control DSS mice (Figure 1f). Taken together, these data indicate that NK cells are protective during experimental colitis.

\section{NK cells reduce neutrophil recruitment and neutrophil activation status during DSS-induced colitis}

Given the role of neutrophils in the early response to DSSinduced colitis, and the correlation between acute disease activity in UC and dense influx of neutrophils into the mucosa, and the elevated levels of circulating activated neutrophils during active UC, we next assessed the recruitment and activation status of neutrophils in our model. ${ }^{1,12,20,24}$ DSStreated NK cell-depleted mice contained substantially $(P<0.001)$ more neutrophils in the colon than DSS NK cell-positive mice (Figure 2a,b). Moreover, NK-depleted DSS mice had a significantly higher $(P<0.01)$ percentage of MLN neutrophils expressing the early activation marker CD69, ROS, IL-6, and IL-17A than the DSS-treated controls (Figure 2c-e). Conversely, DSS NK-depleted mice had significantly fewer $(P<0.001)$ IL-10 and IL-4-positive neutrophils than control DSS-treated animals (Figure 2e). Thus, NK cell-mediated protection is associated with a diminished neutrophil recruitment and activation status.

Although neutrophil activation and recruitment is characteristic of acute inflammation and injury in UC and the DSSinduced colitis model, this is not as evident during chronic UC and $\mathrm{T}$-cell transfer models of chronic colitis. Interestingly, previous work in $\mathrm{CD} 45 \mathrm{RB}^{\text {high }}$ transfer models, which represent a pathogenic T-cell-driven chronic model of colitis, has indicated that NK cells may also regulate $\mathrm{T}$-cell populations. ${ }^{13,14}$ Thus, we investigated the potential influence of NK cells on T-cell populations during DSS colitis (Supplementary Figure S4). Although MLN and colonic T-cell numbers did not 

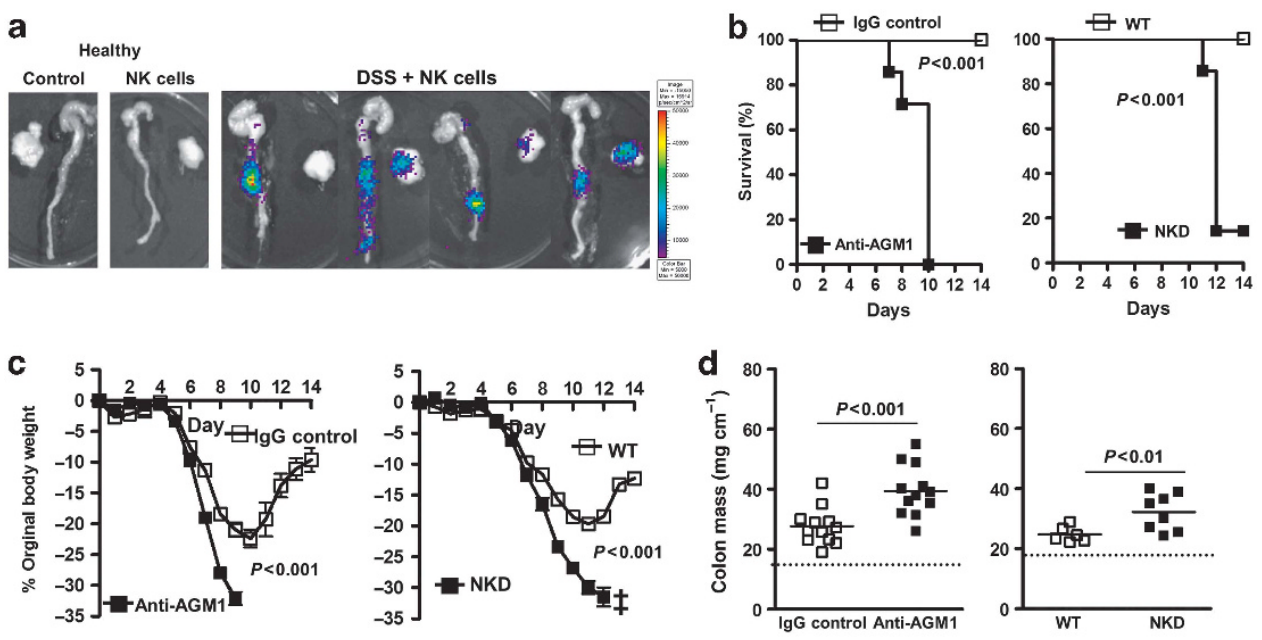

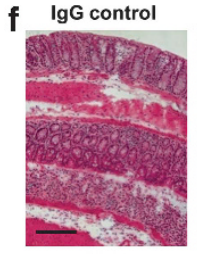

WT
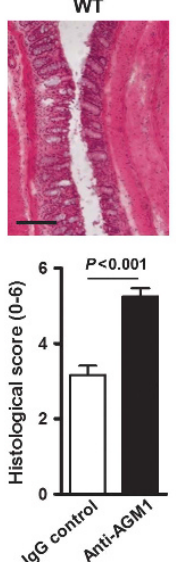

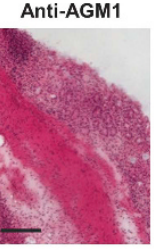

NKD
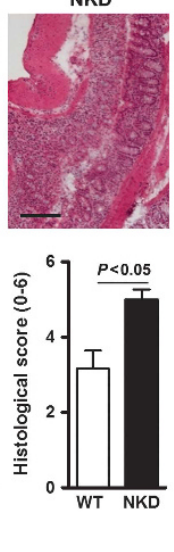
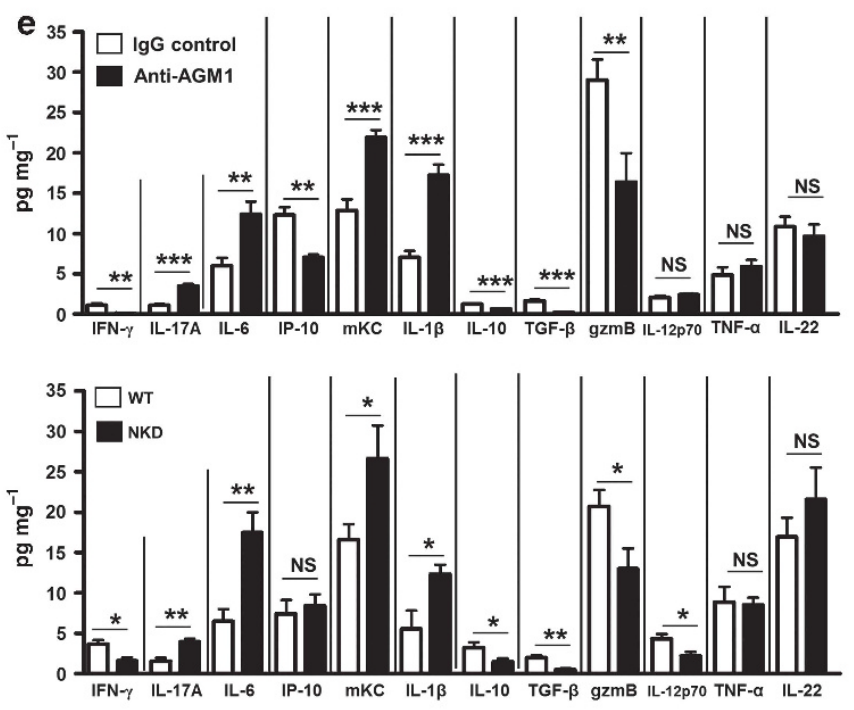

Figure 1 Natural killer (NK) cell-deficient mice rapidly succumb to detran sodium sulfate (DSS)-induced colitis. (a) Bioluminescent NK cell trafficking $22 \mathrm{~h}$ post-transfer in mice with DSS-induced colitis. NK cells were isolated from the spleens and lymph nodes of FVB/N transgenic $\beta$-actin-luciferase mice 7 days after DSS administration. Cells ( $\sim \times 10^{6}$ cells/mouse) were injected intravenously into the tail veins of healthy or DSS-induced recipients $(n=6)$. (b) Mortality, (c) body weight, and (d) colon mass in two models with NK cell deficiency; NK cell depletion using anti-asialo GM1 (anti-AGM1; every 3-4 days) or NK cell-deficient (NKD) mice (plus control mice) in C57BL/6 mice undergoing DSS-induced colitis. $\ddagger$ indicates one animal remaining. Dotted line indicates average healthy colon mass. (e) Cytokine levels in whole colonic homogenates on day 7 DSS. (f) Representative histology of hematoxylin and eosin-stained colon sections at day 7 DSS. Bar $=200 \mu \mathrm{m}$. Graphs represent overall histology score. Data are means \pm s.d. $n=7-12$ mice per group, representative of three experiments. ${ }^{\star} P<0.05$, ${ }^{\star *} P<0.01$, and ${ }^{\star \star *} P<0.001$. gzmB, granzyme B; IFN, interferon; IgG, immunoglobulin G; IL, interleukin; mKC, mouse keratinocyte-derived chemokine; NS, not significant; TGF, transforming growth factor; WT, wild type.

alter $(P>0.05)$ between DSS NK cell-depleted mice and controls, we did observe significantly higher expression $(P<0.05)$ of the early activation antigen, CD69, in tandem with inflammatory cytokine expression, including IFN- $\gamma$ and IL-17A (Supplementary Figure S4). These data indicate that NK cells may also act to regulate the activation of T-cell responses during acute DSS-induced colitis.

\section{NK cells directly modulate neutrophil responses}

We next investigated whether the NK cell-protective effect was mediated via a direct interaction between NK cells and neutrophils. ${ }^{3}$ Colonic sections from control DSS-treated mice revealed augmented levels of NK cells and neutrophils in close proximity as well as in direct contact, contrary to healthy mice, where contact and numbers of these cells were very low (Figure 3a). We then isolated both healthy naive and DSSactivated NK cells and performed both direct and transwell coculture experiments with in vivo activated neutrophils. Both naive and DSS NK cells significantly lowered $(P<0.001)$ the percentage of apoptotic (annexin $\mathrm{V}^{+}$) neutrophils when compared with neutrophils cultured alone (Figure $\mathbf{3 b}$ ). This NK cell-mediated pro-survival effect was reduced somewhat 

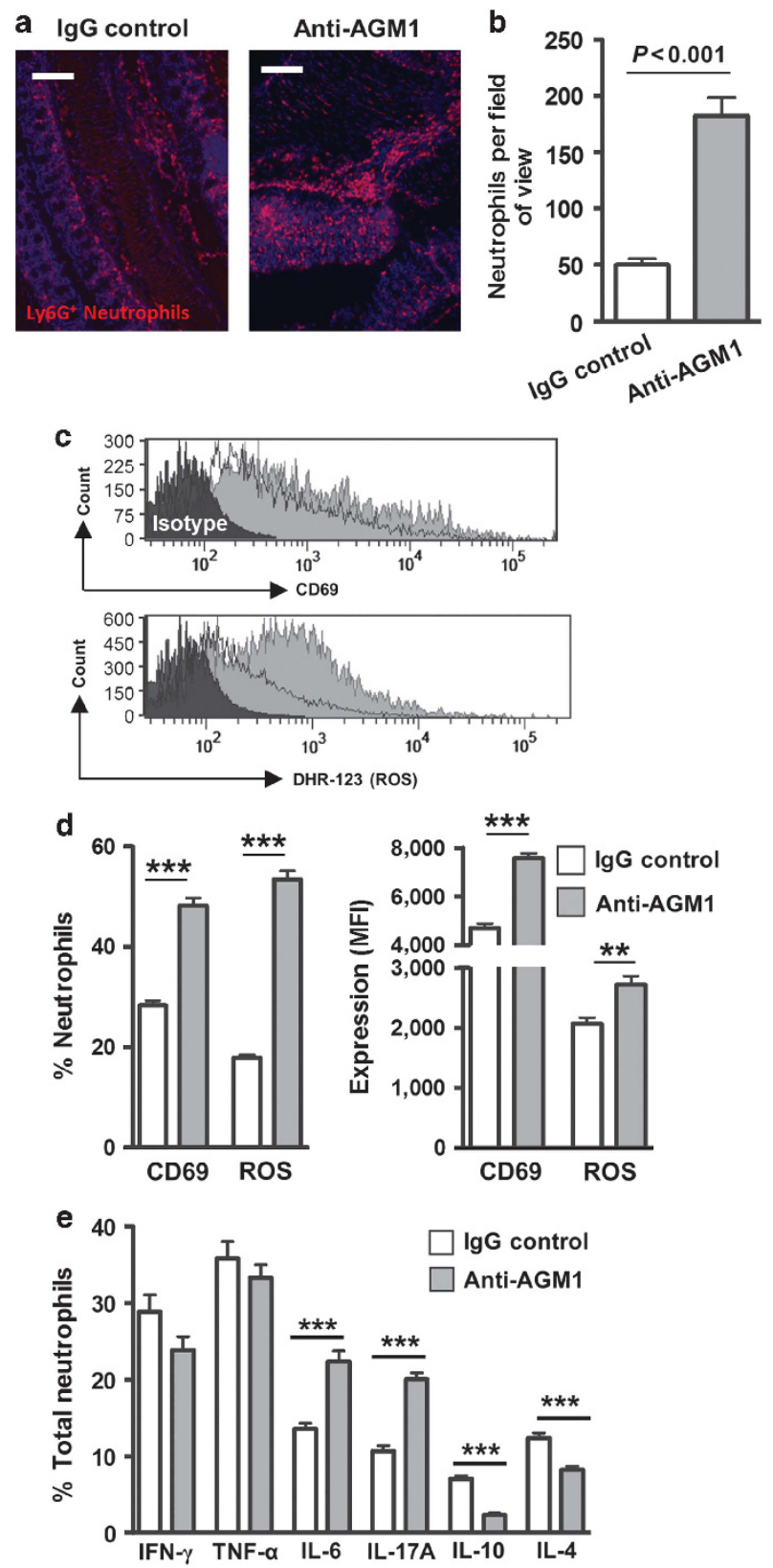

Figure 2 Natural killer (NK) cells reduce neutrophil recruitment and neutrophil activation status during detran sodium sulfate (DSS)-induced colitis. (a) Immunofluorescent staining of neutrophils (Ly6G, red) and nuclei (blue) from DSS control (NK cell ${ }^{+}$) and NK-depleted mice (NK cell- ${ }^{-}$. A representative picture for each group $(n=4)$ is shown. Bar $=200 \mu \mathrm{m}$. (b) Bars represent means \pm s.d. of the total number of positive cells per field of view. Flow cytometric analysis of CD69 and reactive oxygen species (ROS; DHR-123) neutrophil responses from DSS $\mathrm{NK}$ cell ${ }^{+}$or NK cell ${ }^{-}$mice. (c) Black outline represents $\mathrm{NK}^{-}$cell ${ }^{+}$and filled gray represents $\mathrm{NK}^{-}$cell ${ }^{-}$on histograms. (d) Columns represent the percentage of $\mathrm{Ly} 6 \mathrm{G}^{+}$cells expressing marker mean fluorescent intensity $(\mathrm{MFI}) \pm$ s.d. Cytokine profiles of neutrophils from DSS NK cell ${ }^{+}$or NK cell ${ }^{-}$mice. Cells were stimulated for $6 \mathrm{~h}$ with $\mathrm{BD}$ Leukocyte Activation Cocktail plus BD GolgiPlug, stained with Ly6G, permeabilised, and stained with anti-cytokine flurochrome monoclonal antibody. (e) Data represent percentage of cytokine positive neutrophils out of the total neutrophil population. Data are means \pm s.d., $n=12$ mice per group, representative of three experiments. ${ }^{*} P<0.05,{ }^{* *} P<0.01$, and ${ }^{\star \star \star} P<0.001$. AGM1, asialo GM1; IFN, interferon; IgG, immunoglobulin G; $\mathrm{IL}$, interleukin; TNF, tumor necrosis factor. within the transwell system, suggesting a requirement for direct cell-to-cell contact (Figure 3b). This suggests that the NK cellinhibitory response is not simply due to removal of neutrophils via induction of apoptosis and is in agreement with recent human studies. ${ }^{25,26}$

As an increase in neutrophils, neutrophil-derived oxidative stress molecules (ROS, inducible nitric oxide synthase), and pro-inflammatory cytokines (IL-6, IL-17) directly correlates with clinical disease activity and epithelial pathology in experimental colitis models and increases in acute disease activity in $\mathrm{UC},{ }^{27,28}$ we analyzed the effect of NK cells on these readouts of neutrophil function. DSSactivated NK cells were capable of significantly reducing $(P<0.001)$ the percentage of $\mathrm{ROS}^{+}$neutrophils in co-culture. This downregulation of ROS by NK cells was also apparent within the transwell system $(P<0.01)$ when compared with neutrophils cultured alone (Figure 3c). A significant $(P<0.01)$ inhibitory effect by DSS-NK cells on inducible nitric oxide synthase production was also detected in the co-cultures but was absent $(P>0.05)$ in the transwell system (Supplementary Figure S5). Neutrophil cytokine profiles from co-cultures revealed that DSS activated but not naive NK cells were able to significantly reduce $(P<0.001)$ both the percentage and total production of IL- 6 from neutrophils. When NK cells and neutrophils were separated by the transwell membrane, IL-6 production was restored (Figure 3d). Significant NK cell inhibition $(P<0.05)$ of IL17 neutrophil responses was also apparent (Supplementary Figure S5). By contrast, the percentage of IL- $10^{+}$neutrophils, as well as total IL-10 production, were significantly increased $(P<0.001)$ in the DSS-activated NK cell-neutrophil cocultures. This positive effect of DSS NK cells on neutrophil IL-10 production was then negated $(P>0.05)$ within the transwell system (Figure 3e). This suggests that IL-6, IL-17, and IL-10 modulation is largely dependent on direct cell-to-cell contact. Thus, NK cells alter the inflammatory phenotype of neutrophils and is largely dependent on direct NK cellneutrophil contact and is suggestive of a receptor-ligandmediated mechanism of action.

\section{NK cell-neutrophil interaction is mediated via NKG2A receptor in vitro}

To pursue this possibility further, we investigated the potential involvement of an NK cell receptor modulating neutrophil function as NK cells express a wide variety of receptors on their surface. ${ }^{1}$ During DSS-induced colitis, NK cells significantly downregulated $(P<0.01)$ expression of both NKp46 and KLRG1 when compared with healthy NK cells. Conversely, substantial upregulation $(P<0.01)$ of NKG2A and NKG2D was apparent on DSS NK cells, when compared with controls (Figure 4a,b). Neutralisation of the NKG2A receptor with a blocking antibody in the co-culture system negated the inhibitory effect of NK cells on neutrophil activity by significantly increasing $(P<0.001)$ the percentage of ROS ${ }^{+}$ and IL- $6^{+}$neutrophils when compared with controls (Figure 4c,d). IL-17 expression also appeared to be partly 
a Healthy control

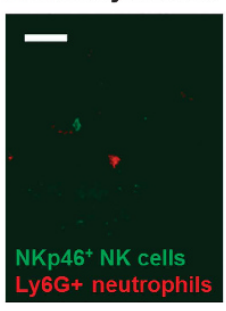

b

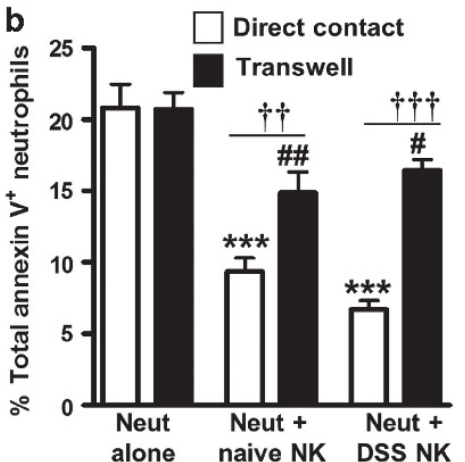

alone naive NK DSS NK

d

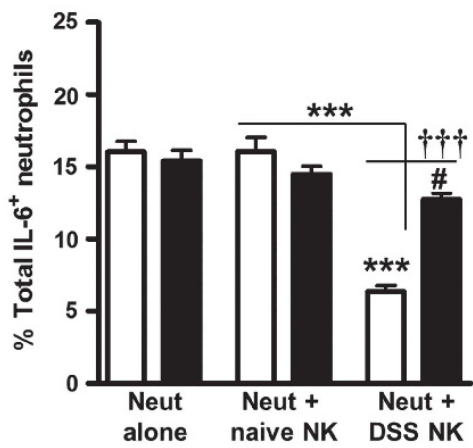

IL-10 percentage

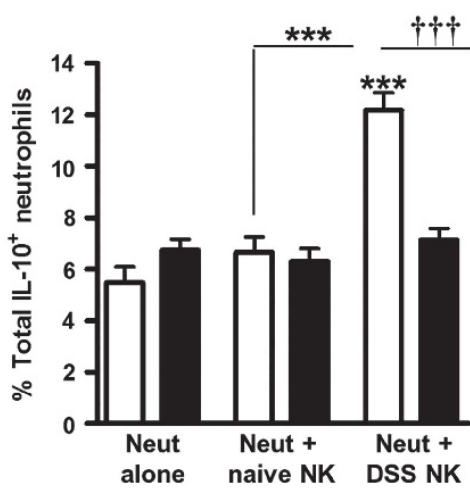

D3
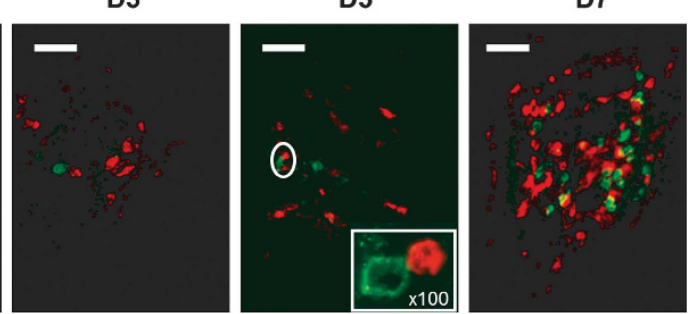

c

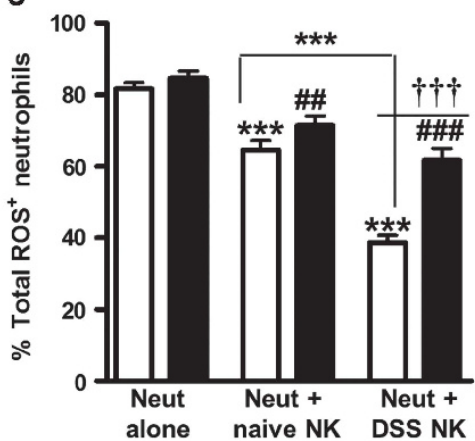

IL-6 total levels

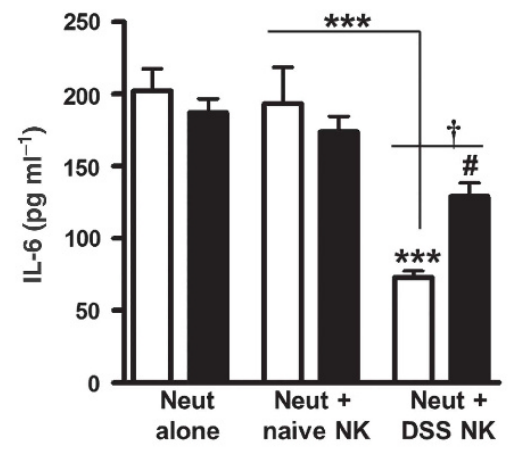

IL-10 total levels

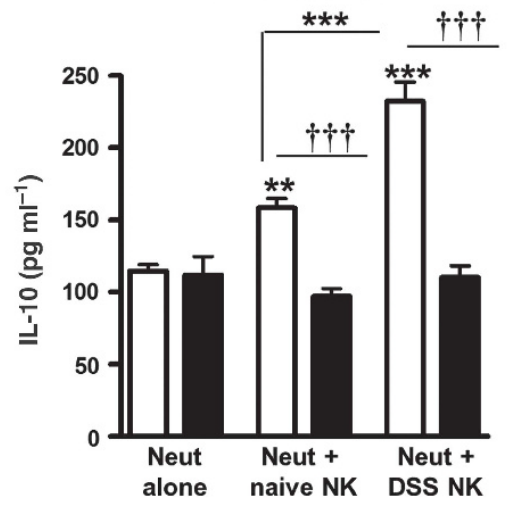

Figure 3 Natural killer (NK) cells directly modulate neutrophil (Neut) responses. (a) Immunofluorescent staining of neutrophils (Ly6G, red) and NK cells (NKp46, green) from healthy and detran sodium sulfate (DSS) mice. A representative picture for each group $(n=4)$ is shown. D1-day 1 post-DSS. $\mathrm{Bar}=60 \mu \mathrm{m}$. (b-e) Co-culture studies examining NK cell-neutrophil responses. Neutrophils were isolated from the peritoneum of mice intraperitoneally injected with $4 \%$ thioglycolate after $8 \mathrm{~h}$. NK cells were also isolated from either healthy control mice (naive NK cells) or from mice 7 days post-DSS (DSS NK cells). These cells were either cultured in direct contact or within transwells at a ratio of $5: 1$ neutrophils:NK cells for $6 \mathrm{~h}$. (b) Columns represent the percentage of Ly6G ${ }^{+}$cells expressing annexin $\mathrm{V} \pm \mathrm{s}$.d. (c) Cultured neutrophils were incubated with DHR-123 before addition of phorbyl myristate acetate, cells were then analyzed by flow cytometry to determine the percentage of DHR-123/ROS (reactive oxygen species)-positive cells. (d, e) Cells were stimulated for $6 \mathrm{~h}$ with BD Leukocyte Activation Cocktail plus BD GolgiPlug, stained with Ly6G, permeabilised and stained with anti-cytokine flurochrome monoclonal antibody. Data represent percentage of cytokine-positive neutrophils out of the total neutrophil population. Supernatants from co-cultures were also analyzed for total production of annotated cytokine. Data are means \pm s.d., $n=10$, representative of three experiments. ${ }^{* *} P<0.01$ and ${ }^{* * *} P<0.001$ for differences between direct co-culture conditions, ${ }^{\sharp} P<0.05$, ${ }^{\# \#} P<0.01$, and ${ }^{\# \# \#} P<0.001$ indicates differences between transwell conditions and ${ }^{\dagger} P<0.05,{ }^{\dagger \dagger} P<0.01$, and ${ }^{\dagger \dagger} P<0.001$ shows differences between the co-cultures and transwells. IL, interleukin. 
a
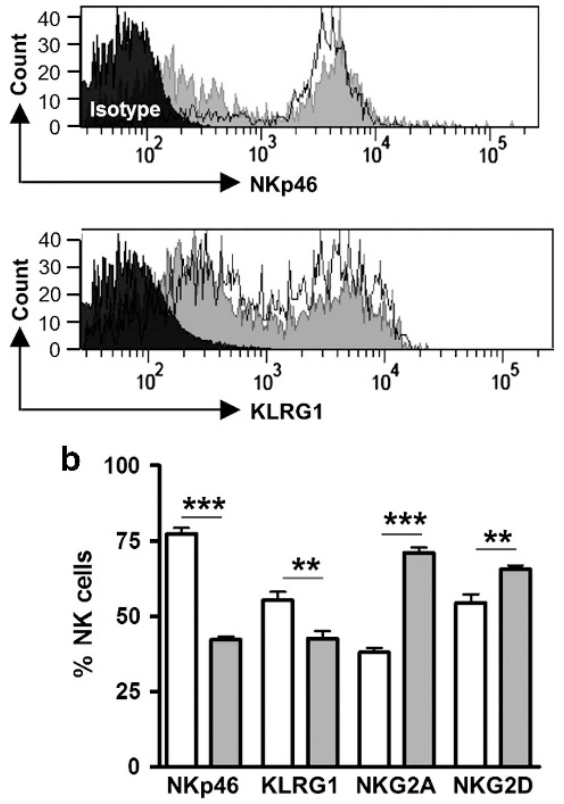

C
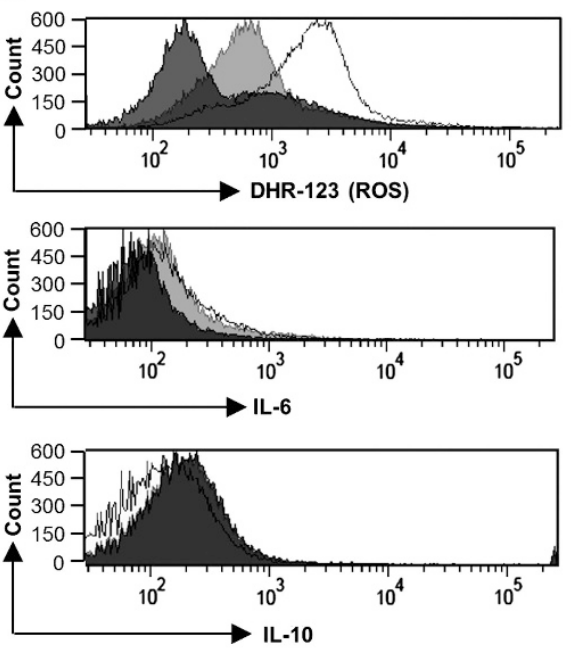
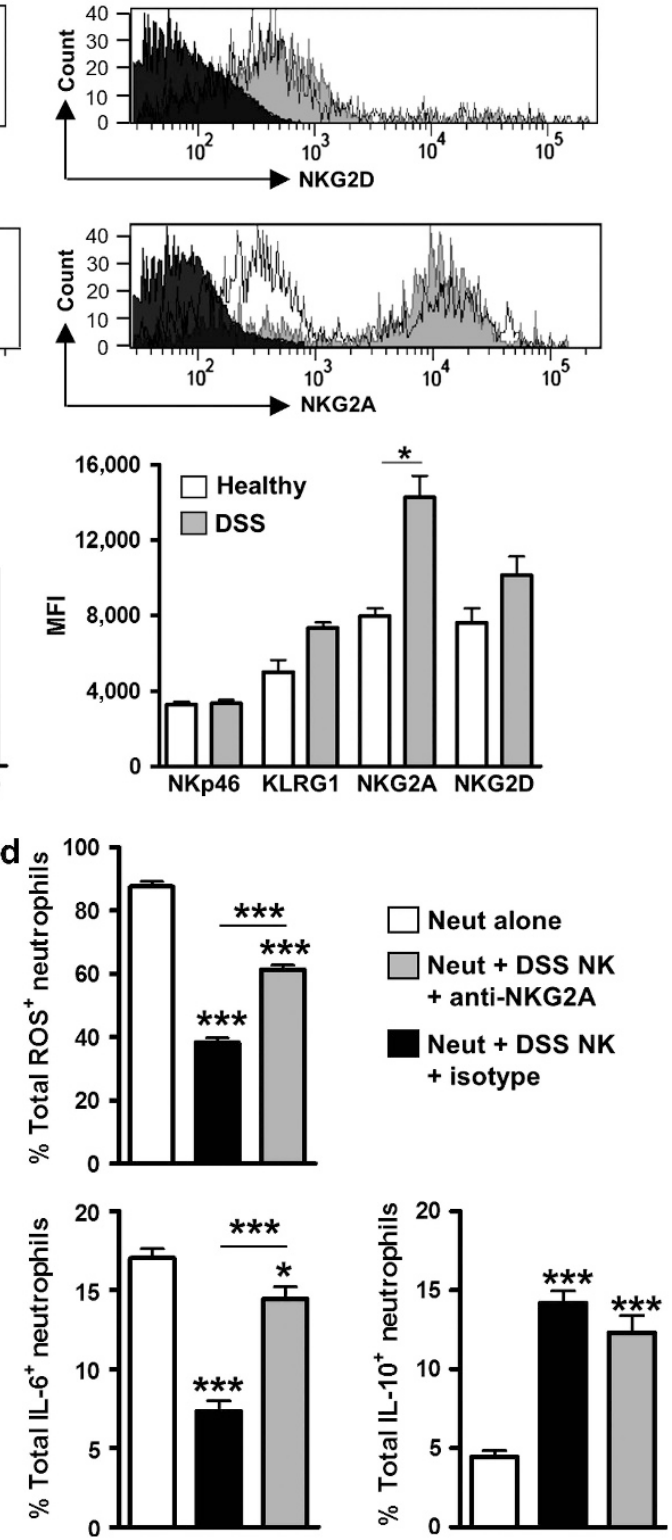

Figure 4 Natural killer (NK) cell-neutrophil interaction is mediated via NKG2A receptor in vitro. Flow cytometric analysis of NK cell-receptor expression in mesenteric lymph nodes NK cells isolated from both healthy control or from mice day at 7 days post-DSS (detran sodium sulfate). (a) On histograms, black outline represents healthy and filled gray represents DSS NK cells. (b) Bar graphs represent the percentage and mean fluorescent intensity (MFI) of NK cells expressing markers \pm s.d.. Neutrophil and DSS NK cells were co-cultured in the presence of $30 \mu \mathrm{gl}^{-1}$ anti-NKG2A or isotype control monoclonal antibody for $6 \mathrm{~h}$, two independent experiments. (c) On histograms, black outline represents neutrophils cultured alone, filled gray represents neutrophils plus DSS NK cells plus anti-NKG2A, and black filled represents neutrophil plus DSS NK cells plus isotype control. (d) Bar graphs represent the percentage of neutrophils expressing inflammatory molecules \pm s.d. $n=6$, two independent experiments. ${ }^{*} P<0.05,{ }^{* \star} P<0.01$, and ${ }^{* * *} P<0.001$.

IL, interleukin; ROS, reactive oxygen species.

controlled $(P<0.05)$ by NKG2A (Supplementary Figure S6). Notably, the increased expression of IL-10 by neutrophils was unaffected $(P>0.05)$ by neutralisation of NKG2A, suggesting other NK cell receptors have a role in this effect (Figure $4 \mathbf{c}, \mathbf{d}$ ). Co-incubation of NK cells and neutrophils with anti-NKG2D did not significantly affect $(P>0.05)$ any of the neutrophil readouts tested. These studies indicate that NK cells may directly modulate various neutrophil inflammatory functions via their inhibitory receptor NKG2A and other additional NK receptors that remain to be identified.
Blockade of NKG2A in vivo exacerbates disease severity during DSS-induced colitis and corresponding neutrophil inflammatory responses

To further confirm the involvement of the inhibitory NK cell receptor NKG2A in regulating acute inflammation during DSSinduced colitis, mice were administered with anti-NKG2A to actively block NKG2A receptor function in vivo (Figure 5). Treatment of mice with anti-NKG2A led to $100 \%$ mortality $(P<0.001) 9$ days into acute experimental colitis, which was also observed within the anti-AGM1-treated group. As shown 

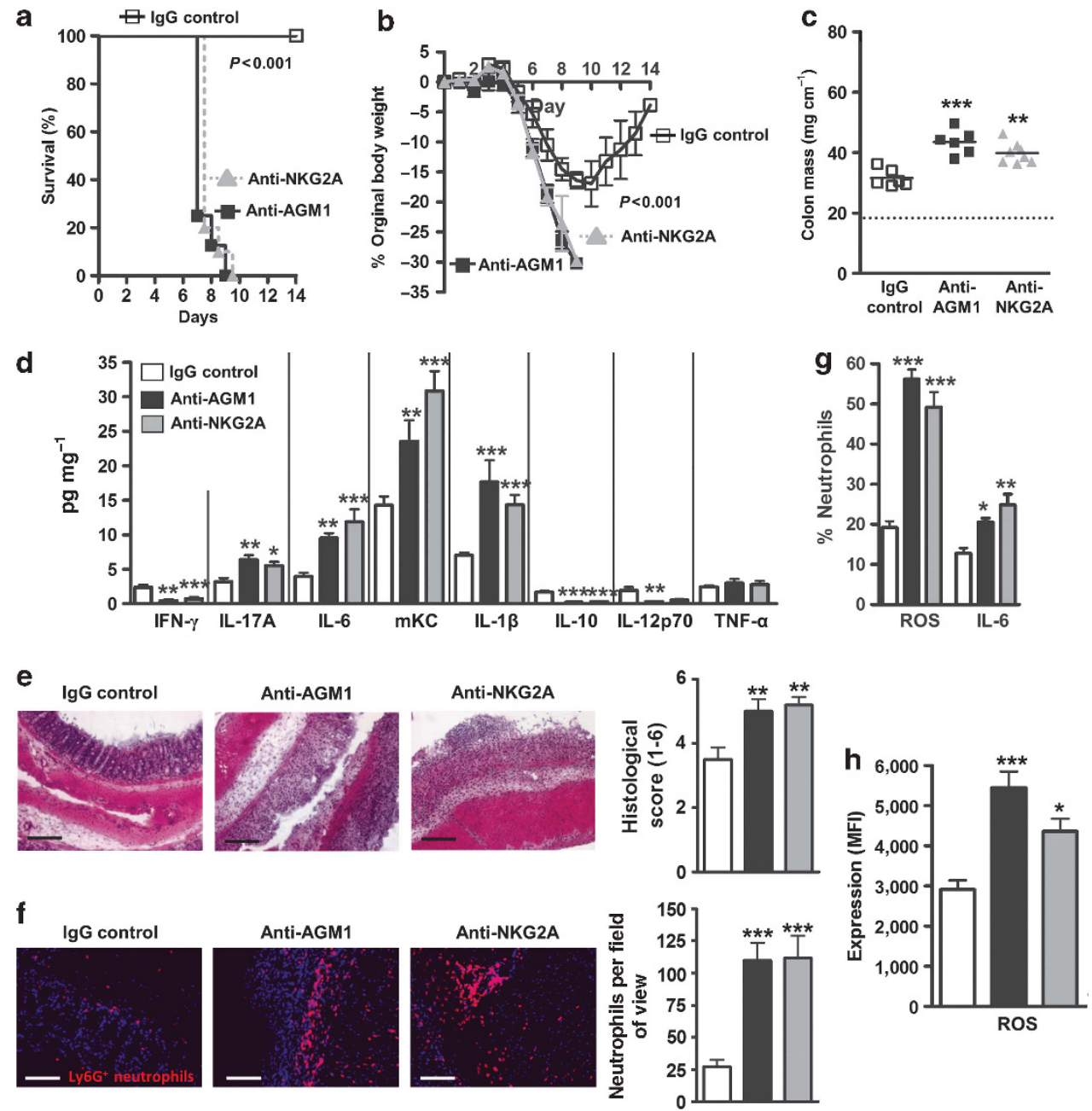

Figure 5 Blockade of NKG2A in vivo exacerbates disease severity during detran sodium sulfate (DSS)-induced colitis and corresponding neutrophil inflammatory responses. (a) Mortality, (b) body weight loss, and (c) colon mass in mice treated with anti-NKG2A or anti-asialo GM1 (anti-AGM1; every 34 days), (plus isotype immunoglobulin G (IgG) control mice) in mice undergoing DSS-induced colitis. Dotted line indicates average healthy colon mass. (d) Cytokine levels in whole colonic homogenates on day 7 post-DSS. (e) Representative histology of hematoxylin and eosin-stained colon sections at day 7 post-DSS (Bar $=200 \mu \mathrm{m}$ ) and graph shows overall histology score. (f) Immunofluorescent staining of neutrophils (Ly6G, red) and nuclei (blue) from DSS control (natural killer (NK) cell ${ }^{+}$), NK-depleted mice (anti-AGM1) and anti-NKG2A mice. A representative picture for each group $(n=4)$ is shown. $\mathrm{Bar}=100 \mu \mathrm{m}$. Bars represent means $\pm \mathrm{s}$.d. of the total number of positive cells per field of view. Flow cytometric analysis of reactive oxygen species (ROS; DHR-123) and interleukin (IL)-6 neutrophil responses. Bar graphs represent the (g) percentage and (h) mean fluorescent intensity of neutrophils expressing markers \pm s.d. Data are means \pm s.d., $n=8-10$ mice per group. ${ }^{\star} P<0.05,{ }^{\star \star} P<0.01$, and ${ }^{\star \star \star} P<0.001$. IFN, interferon; mKC, mouse keratinocyte-derived chemokine; TNF, tumor necrosis factor.

in Figure $1 \mathbf{b}$, IgG control (NK cell ${ }^{+} / \mathrm{NKG}_{2} \mathrm{~A}^{+}$) mice survived until the end of the study (Figure 5a). Body weight loss and colon weight were significantly greater $(P<0.001)$ in both antiNKG2A and anti-AGM1-treated mice compared with IgG control DSS mice (Figure $\mathbf{5 b}, \mathbf{c}$ ). Analysis of colonic cytokines revealed that both NK-depleted and anti-NKG2A-treated mice produced significantly greater $(P<0.01)$ levels of IL-17A, IL-6, mKC, and IL-1 $\beta$ but reduced $(P<0.05)$ IFN- $\gamma$ and IL-10 compared with control IgG DSS animals (Figure 5d). In addition, both anti-NKG2A and anti-AGM1-treated mice had heightened colonic pathology compared with IgG control DSS mice (Figure 5e).

Further analysis of neutrophil responses in anti-NKG2Atreated mice revealed significantly increased colonic neutrophil recruitment in tandem with greater ROS and IL-6 expression in
MLN neutrophils, similar to NK cell-depleted mice and in comparison to DSS IgG control mice (Figure $\mathbf{5 f}-\mathbf{h}$ ). These data confirm that the NK cell-inhibitory receptor NKG2A is directly involved in the downregulation of inflammatory neutrophil functions during acute colitis.

\section{DISCUSSION}

NK cells have a significant role linking the innate and adaptive arms of the immune system but their role during colitis remains elusive. Here we report that NK cells can downregulate neutrophil function, preventing severe and ultimately fatal acute colitis in an experimental model. NK cells can reduce neutrophil recruitment and modulate their pro-inflammatory activation status, through direct cell-to-cell contact via the 
NKG2A receptor thereby providing a protective effect during DSS-induced colitis (Figure 6).

During UC, disruption of the epithelial barrier causes substances from the gut lumen, including the microflora, to trigger activation of innate immune populations. Initiation of experimental colitis, through oral administration of DSS, compromises gut barrier function, resulting in a strong inflammatory response with consequent crypt destruction, mucosal erosion, and ulcerations, which resembles a number of features similar to those seen in acute active UC. Utilizing luciferase expressing NK cells revealed direct trafficking of NK cells into the inflamed colon during DSS-induced colitis. The considerable NK cell recruitment, in tandem with strong expression of several pro-inflammatory factors, suggested that removal of NK cells would be associated with a positive impact on the overall health of animals with colitis. On the contrary, using two models of NK cell deficiency, we observed that these mice were significantly more susceptible to weight loss, diarrhea, pro-inflammatory cytokine levels, and ultimately mortality during the acute phase of disease, indicating a key role for NK cells in protection against DSS-induced colitis. Previously, a protective role for NK cells has been reported in the IL- $10^{-/-}$transfer model, where depletion of NK cells led to acceleration of disease. ${ }^{13}$ Notably, emerging evidence indicates that NK cells can also have a critical protective role in a number of other immune-mediated diseases. For example, in the nonobese diabetic mouse model of Type 1 diabetes, prevention of diabetes by polyinosinic-polycytidylic acid was associated with the formation of Th2 (T helper type 2)-enriched environment in the spleen and pancreas and was a result of NK cell function in response to islet autoantigens. ${ }^{5}$ Furthermore, in a mouse model of multiple sclerosis, enrichment of NK cells resulted in disease amelioration through NK cell-associated suppression of myelin-reactive Th17 cells. ${ }^{6}$ Thus, it appears that NK cells are critical for preventing uncontrolled inflammation in an intestinal setting and this may correlate with their function in other organs under specific disease settings.

As previously mentioned, NK cells can have a protective or pathogenic role in inflammatory diseases. This can be a direct consequence of NK cell function or through interaction with various components of the immune system to thereby limit or exacerbate immune responses. Removal of NK cells resulted in a considerable influx of neutrophils into the inflamed colon during DSS-induced colitis. In addition, MLN neutrophils were

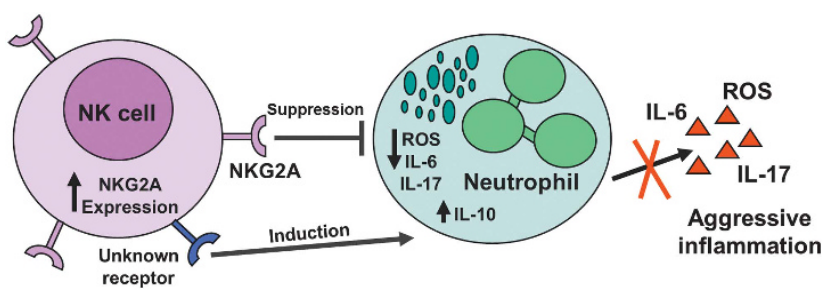

Figure 6 Schematic representation of the mechanism by which natural killer (NK) cells downregulate neutrophil function during experimental colitis. IL, interleukin; ROS, reactive oxygen species. in a hyper-activated state, as shown by increased CD69, ROS, IL-6, and IL-17A expression levels. Neutrophils, like NK cells, are an important cellular innate immune population that are rapidly recruited to sites of inflammation. Upon activation, these phagocytic cells produce a wide range of factors involved in host defense, including ROS, anti-bacterial peptides, and protease-containing granules. Recent studies have also shown that in response to cytokines or pathogen products, they can produce both pro- and anti-inflammatory cytokines/chemokines and therefore can have an important role in regulating immune responses. ${ }^{29}$ Notably, infiltration of neutrophils is a striking histological feature in the lesions of active UC and experimental colitis models. ${ }^{20,28}$ Interestingly, both IL-6 and IL-17 are known inducers of neutrophil chemoattractant molecules, ${ }^{29}$ therefore the significant influx we observe within NKD animals may be a result of a cytokinedriven positive-feedback loop further perpetuating recruitment of neutrophils. In UC patients with active disease and in rodent experimental colitis models, these neutrophils are activated in the colonic mucosa and release their toxic oxidative metabolites, which are associated with mucosal damage. ${ }^{30,31}$ Also, IL- 6 and IL-1 $\beta$ are potent neutrophil activators; therefore increased production of these cytokines would further enhance the damaging ROS phenotype of neutrophils leading to severe epithelial degeneration. Indeed, a recent study using a TKTL1 ${ }^{-1-}$ mice, which are defective in antioxidants, showed more severe inflammation in response to DSS when compared with wild-type animals. ${ }^{31}$ Therefore, during colitis in mice, and possibly human UC, NK cells can have a protective role by modulating various inflammatory aspects of neutrophil function.

In addition to their apparent regulatory action on neutrophils, it also appears that NK cells may act to modulate T-cell responses. Although overall T-cell numbers were not altered, their inflammatory profile was elevated in NK cell-depleted mice, possibly contributing to the enhanced overall colonic inflammatory cytokine levels (particularly IL-17A) observed during colitis. Currently, we cannot differentiate if this increase is a direct response to the depletion of NK cells or as a result of other factor(s). Previously, we have shown T-cell-activation status is significantly elevated from day 8 onwards during DSSinduced colitis. This is considerably later than the time we begin to observe mortality (from day 6) within colitic NK celldepleted mice. ${ }^{20}$ Therefore, it would not be expected that $\mathrm{T}$ cells would have a significant detrimental role in the earlier stages (days 1-6) of this acute colitis model. Importantly, previous and recent data using T-cell-reconstituted $\mathrm{RAG}^{-1-}$ models have indicated that NK cells have a key role in regulating colitogenic T-cell responses during chronic colitis. ${ }^{13,14}$ Furthermore, in a mouse model of multiple sclerosis, enrichment of NK cells resulted in disease amelioration through NK cell-associated suppression of myelin-reactive Th17 cells. ${ }^{6}$ Our data, along with these other published studies, suggest that in addition to their critical early protective and regulatory effect on neutrophil function and acute disease, NK cells may also regulate T-cell responses during chronic inflammation. Additional studies are 
required to properly dissect the contribution of NK-T cell interactions to chronic inflammation.

The capacity of NK cells to interact and modulate neutrophil function in vitro has been assessed both in rodent and human cells; however, the relevance of this in vivo remains largely unexplored. ${ }^{3}$ To identify the mechanisms governing these responses in vivo, we isolated activated neutrophils and NK cells from mice with DSS-induced colitis. From direct and transwell co-culture studies, downregulation of neutrophil proinflammatory responses, including IL-6, IL-17, and ROS, is mediated, chiefly, through direct cell-to-cell contact. This also appears to be the case for induction of the anti-inflammatory cytokine IL-10 by neutrophils. During DSS-induced colitis, we observed an expansion of the $\mathrm{NKG}_{2} \mathrm{~A}^{+} \mathrm{NK}$ cell population. NKG2A is an inhibitory C-type lectin most commonly found on NK cells and some cytotoxic $\mathrm{CD} 8{ }^{+} \mathrm{T}$ cells. The receptorligand binding of NKG2A to the MHC class Ib antigen HLA-E (human leukocyte antigen E; Qa-1b in mice) initiates a signaling cascade downstream of the NKG2A immunoreceptor tyrosine-based inhibition motif that suppresses effector cell cytotoxic activity. ${ }^{32}$ Notably, when we blocked this receptor in vitro, we restored the inflammatory phenotype of activated neutrophils indicating a role for this inhibitory NK cell receptor on neutrophil function. However, expression of IL-10 was unaffected, suggesting that other NK cell receptors have a role in this pathway. Subsequent in vivo studies showed that blockade of the NKG2A receptor gave a similar disease phenotype as seen with whole NK cell depletion, confirming that this specific receptor has a direct protective role during acute colitis through regulation of inflammatory neutrophil function. We show here, for the first time, a direct receptorligand interaction between NK cells and neutrophils that acts as a switch to downregulate a detrimental inflammatory neutrophilic response. Importantly, this inhibitory response is not mediated through removal of neutrophils via induction of apoptosis as we actually observed prolonged survival of neutrophils when in co-culture with NK cells. ${ }^{3}$

Anti-NKG2A-treated mice also have excessive neutrophil accumulation, suggesting that direct cell-to-cell contact between $\mathrm{NKG}_{2} \mathrm{~A}^{+}$NK cells and neutrophils may also have a role in the modulation of colonic neutrophil infiltration. NKG2A may serve to prevent or reduce an inflammatory neutrophil-driven positive-feedback loop, via inhibition of cytokines (such as IL-6 and IL-17), subsequently impacting on overall neutrophil recruitment. Early release of NK-derived soluble factors may also have an inhibitory role in initial neutrophil recruitment during colitis. Previously, IFN- $\gamma$ has been shown to induce monopoiesis (differentiation of monocytes) and inhibit neutrophil development during inflammation. ${ }^{33}$ Indeed, IFN $-\gamma^{-1-}$ mice have increased neutrophil production and recruitment during inflammation, ${ }^{33}$ with IFN$\gamma$ directly able to inhibit pathogenic neutrophil accumulation via blockade of IL-17 and other neutrophil-prompting chemokines/cytokines. ${ }^{34}$ IL-10 is also known to regulate neutrophil recruitment by attenuating chemokine (mKC) production, resulting in inhibition of neutrophil trafficking into local mucosal tissues. ${ }^{35,36}$ Correspondingly, we observed increased levels of neutrophil recruitment-inducing chemokines (mKC) and cytokines (IL-17, IL-6, and IL-1 $\beta$ ) in colonic homogenates of NK cell-depleted mice. This may correlate with the loss of NK cell-specific IFN- $\gamma$ and IL-10 production, resulting in the excessive neutrophil accumulation observed during colitis in NK cell-depleted mice. Thus, the absence of NKG2A-expressing NK cells significantly alters the inflammatory microenvironment promoting the excessive influx of neutrophils. Further studies, focusing on NKG2A and NK cellderived soluble factors, will determine their relative involvement in the context of colitis and neutrophil recruitment.

The effect of NK cells on neutrophils in the human inflammatory setting is currently unclear. Although NK cell numbers in UC patients appear to vary, functional studies have indicated normal NK cell activity in patients with inactive disease but pronounced defects in cytotoxic responses in those with active UC disease. ${ }^{16,17}$ Importantly, clinical change from active to inactive disease are associated with enhancement of NK activity. ${ }^{18}$ Furthermore, freshly isolated NK cells from active UC patients, although initially defective in lytic functions, can become cytotoxic after in vitro activation, suggesting no major maturational defects in NK precursor cells. ${ }^{18}$ More recent data have indicated that another NK cellinhibitory receptor, KIR2DL2/3, is genetically associated with Crohn's disease and other chronic inflammatory diseases in the presence of its cognate ligand HLA-C1. ${ }^{37}$ These data point to a number of possible scenarios in the clinical disease settingreduced NK cell-receptor expression (such as NKG2A), rather than complete loss, during active disease, or specific genetic polymorphisms that subsequently increases the risk of disease development. Future studies are required to properly dissect these genetic associations and to determine if this NK cellprotective mechanism is present in the patients with active inflammation.

In conclusion, our findings not only identify a previously unknown immunoregulatory mechanism for NK cells during acute inflammation but also identify a specific NK cell receptor-NKG2A-that can directly regulate neutrophil function. This may open a new promising therapeutic approach for intestinal diseases such as UC, where neutrophil infiltration is a predominant feature.

\section{METHODS}

Mice and in vivo studies. Mice (C57BL/6JOlaHsD and $\mathrm{FVB} / \mathrm{N})$ were used at 12-14 weeks of age. Animal husbandry and experimental procedures were approved by the University College Cork Animal Experimentation Ethics Committee. Mice received intraperitoneal injections (every 3-4 days) of either anti-AGM1 or anti-NKG2A to deplete NK cells/block receptor function. ${ }^{4}$ Mice were given 3\% DSS (45 kDa; TdB Consultancy, Uppsala, Sweden) ad libitum in their drinking water for 6 days followed by water for the remainder of the study as described. ${ }^{19,20}$

Bioluminescent imaging. NK cells were isolated and adoptively transferred into $\mathrm{FVB} / \mathrm{N}$ recipients with DSS-induced colitis and imaged $22 \mathrm{~h}$ post-transfer as described in Supplementary Methods. 
Flow cytometry and oxidative burst analysis. Single-cell suspensions were prepared and stained as previously described and in Supplementary Methods. ${ }^{4}$ For intracellular staining, cells were incubated with BD Activation Cocktail plus GolgiPlug (BD Biosciences, Oxford, UK) for $6 \mathrm{~h}$. Samples were acquired on a FACSLSRII, (BD Biosciences) and data were analyzed using DIVA software (BD Bioscience).

NK cell and neutrophil co-cultures. For detailed information, see Supplementary Methods. NK cells were isolated as described above. Neutrophils were harvested from the peritoneum of mice $8 \mathrm{~h}$ after intraperitoneal injection with $4 \%$ thioglycollate. Direct co- and transwell cultures were then incubated at $37^{\circ} \mathrm{C}$ for the required time frames. For receptor neutralisation studies, cultures were supplemented with $30 \mu \mathrm{g} \mathrm{ml}^{-1}$ of anti-NKG2A, anti-NKG2D, or appropriate isotype control.

Statistical analysis. Experimental results were plotted and analyzed for statistical significance with Prism 4 software (GraphPad Software Inc, La Jolla, CA, USA) using Kaplan-Meier survival curves and logrank test, one-way ANOVA followed by Bonferroni post-hoc correction, and ANOVA Kruskal-Wallis test with Dunn's multiple comparison test. A $P$-value of $<0.05$ was used as significant in all cases.

SUPPLEMENTARY MATERIAL is linked to the online version of the paper at http://www.nature.com/mi

\section{ACKNOWLEDGEMENTS}

The Alimentary Pharmabiotic Center is a research center funded by Science Foundation Ireland (SFI), through the Irish Government's National Development Plan. We and our work are supported in part by SFI grant numbers: 02/CE/B124 and 07/CE/B1368. We thank Professor Wayne Yokoyama, Washington University School of Medicine, St Louis, USA, for the kind gift of NKD mice and critical reading of the manuscript.

\section{AUTHOR CONTRIBUTIONS}

LJH conceived and designed the study, carried out all experiments and analysis and drafted the manuscript. CTM participated in the NK celltrafficking studies, in vitro NK cell-neutrophil studies, and anti-NKG2A in vivo blocking studies. $A Q$ and $G H$ performed animal husbandry, clinical scoring of animals, and participated in processing of tissues. FS secured the funding and helped with drafting of the manuscript. KN and SM conceived and designed the study and helped to draft the manuscript.

\section{DISCLOSURE}

The authors declared no conflict of interest.

c 2013 Society for Mucosal Immunology

\section{REFERENCES}

1. Pegram, H.J., Andrews, D.M., Smyth, M.J., Darcy, P.K. \& Kershaw, M.H. Activating and inhibitory receptors of natural killer cells. Immunol. Cell Biol. 89, 216-224 (2010).

2. Vivier, E. et al. Innate or adaptive immunity? The example of natural killer cells. Science 331, 44-49 (2011).

3. Costantini, C. \& Cassatella, M.A. The defensive alliance between neutrophils and NK cells as a novel arm of innate immunity. J. Leukoc. Biol. 89, 221-233 (2011).

4. Hall, L.J., Clare, S. \& Dougan, G. NK cells influence both innate and adaptive immune responses after mucosal immunization with antigen and mucosal adjuvant. J. Immunol. 184, 4327-4337 (2010).

5. Zhou, R., Wei, H., Tian, Z. \& NK3-like, N.K. cells are involved in protective effect of polyinosinic-polycytidylic acid on type 1 diabetes in nonobese diabetic mice. J. Immunol. 178, 2141-2147 (2007).

6. Hao, J. et al. Central nervous system (CNS)-resident natural killer cells suppress Th17 responses and CNS autoimmune pathology. J. Exp. Med. 207, 1907-1921 (2010).
7. Park, Y.W. et al. Impaired differentiation and cytotoxicity of natural killer cells in systemic lupus erythematosus. Arthritis Rheum. 60, 1753-1763 (2009).

8. Soderstrom, K. et al. Natural killer cells trigger osteoclastogenesis and bone destruction in arthritis. Proc. Natl. Acad. Sci. USA 107, 13028-13033 (2010).

9. Motz, G.T. et al. Chronic cigarette smoke exposure primes NK cell activation in a mouse model of chronic obstructive pulmonary disease. J. Immunol. 184, 4460-4469 (2010).

10. Kaser, A., Zeissig, S. \& Blumberg, R.S. Inflammatory bowel disease. Annu. Rev. Immunol. 28, 573-621 (2010).

11. Hanai, H. et al. Relationship between fecal calprotectin, intestinal inflammation, and peripheral blood neutrophils in patients with active ulcerative colitis. Dig. Dis. Sci. 49, 1438-1443 (2004).

12. D'Odorico, A., D'Inca, R., Mestriner, C., Di Leo, V., Ferronato, A. \& Sturniolo, G.C. Influence of disease site and activity on peripheral neutrophil function in inflammatory bowel disease. Dig. Dis. Sci. 45, 1594-1600 (2000).

13. Fort, M.M., Leach, M.W. \& Rennick, D.M. A role for NK cells as regulators of CD4 + T cells in a transfer model of colitis. J. Immunol. 161, 3256-3261 (1998).

14. Yamaji, O. etal. The development of colitogenic CD4 $(+)$ Tcells is regulated by IL-7 in collaboration with NK cell function in a murine model of colitis. J. Immunol. 188, 2524-2536 (2012).

15. Laroux, F.S. et al. Regulation of chronic colitis in athymic nu/nu (nude) mice. Int. Immunol. 16, 77-89 (2004).

16. Steel, A.W., Mela, C.M., Lindsay, J.O., Gazzard, B.G. \& Goodier, M.R. Increased proportion of CD16(+) NK cells in the colonic lamina propria of inflammatory bowel disease patients, but not after azathioprine treatment. Aliment. Pharmacol. Ther. 33, 115-126 (2011).

17. Shimamoto, M. et al. Selective decrease in colonic CD56(+) T and CD161 (+) Tcells in the inflamed mucosa of patients with ulcerative colitis. World J. Gastroenterol. 13, 5995-6002 (2007).

18. Manzano, L. et al. Functional impairment of natural killer cells in active ulcerative colitis: reversion of the defective natural killer activity by interleukin 2. Gut 33, 246-251 (1992).

19. Melgar, S., Karlsson, A. \& Michaelsson, E. Acute colitis induced by dextran sulfate sodium progresses to chronicity in C57BL/6 but not in BALB/C mice: correlation between symptoms and inflammation. Am. J. Physiol. 288, G1328-G1338 (2005).

20. Hall, L.J., Faivre, E., Quinlan, A., Shanahan, F., Nally, K. \& Melgar, S. Induction and activation of adaptive immune populations during acute and chronic phases of a murine model of experimental colitis. Dig. Dis. Sci. 56, 79-89 (2010).

21. Kim, S., lizuka, K., Aguila, H.L., Weissman, I.L. \& Yokoyama, W.M. In vivo natural killer cell activities revealed by natural killer cell-deficient mice. Proc. Natl. Acad. Sci. USA 97, 2731-2736 (2000).

22. Colonna, M. Interleukin-22-producing natural killer cells and lymphoid tissue inducer-like cells in mucosal immunity. Immunity 31, 15-23 (2009).

23. Wilson, N.J. et al. Development, cytokine profile and function of human interleukin 17-producing helper $\mathrm{T}$ cells. Nat. Immunol. 8, 950-957 (2007).

24. Shea-Donohue, T. et al. Mice deficient in the CXCR2 ligand, CXCL1 (KC/ GRO-alpha), exhibit increased susceptibility to dextran sodium sulfate (DSS)-induced colitis. Innate. Immun. 14, 117-124 (2008).

25. Costantini, C., Micheletti, A., Calzetti, F., Perbellini, O., Pizzolo, G. \& Cassatella, M.A. Neutrophil activation and survival are modulated by interaction with NK cells. Int. Immunol. 22, 827-838 (2010).

26. Bhatnagar, N. et al. Cytokine-activated NK cells inhibit PMN apoptosis and preserve their functional capacity. Blood 116, 1308-1316 (2010).

27. Natsui, M. et al. Selective depletion of neutrophils by a monoclonal antibody, RP-3, suppresses dextran sulphate sodium-induced colitis in rats. J. Gastroenterol. Hepatol. 12, 801-808 (1997).

28. Izzo, R.S., Witkon, K., Chen, A.I., Hadjiyane, C., Weinstein, M.I. \& Pellecchia, C. Interleukin-8 and neutrophil markers in colonic mucosa from patients with ulcerative colitis. Am. J. Gastroenterol. 87, 1447-1452 (1992).

29. Mantovani, A., Cassatella, M.A., Costantini, C. \& Jaillon, S. Neutrophils in the activation and regulation of innate and adaptive immunity. Nat. Rev. 11, 519-531 (2011). 


\section{ARTICLES}

30. Lampinen, M., Sangfelt, P., Taha, Y. \& Carlson, M. Accumulation, activation, and survival of neutrophils in ulcerative colitis: regulation by locally produced factors in the colon and impact of steroid treatment. Int. J. Colorectal Dis. 23, 939-946 (2008).

31. Bentz, S. et al. Lack of transketolase-like (TKTL) 1 aggravates murine experimental colitis. Am. J. Physiol. 300, G598-G607 (2011).

32. Borrego, F., Masilamani, M., Kabat, J., Sanni, T.B. \& Coligan, J.E. The cell biology of the human natural killer cell CD94/NKG2A inhibitory receptor. Mol. Immunol. 42, 485-488 (2005).

33. de Bruin, A.M., Libregts, S.F., Valkhof, M., Boon, L., Touw, I.P. \& Nolte, M.A. IFNgamma induces monopoiesis and inhibits neutrophil development during inflammation. Blood 119, 1543-1554 (2012).
34. Nandi, B. \& Behar, S.M. Regulation of neutrophils by interferon-gamma limits lung inflammation during tuberculosis infection. J. Exp. Med. 208, 2251-2262 (2011).

35. Sun, L., Guo, R.F., Newstead, M.W., Standiford, T.J., Macariola, D.R. \& Shanley, T.P. Effect of IL-10 on neutrophil recruitment and survival after Pseudomonas aeruginosa challenge. Am. J. Respir. Cell Mol. Biol. 41, 76-84 (2009).

36. Jansen, A.M. et al. A Salmonella Typhimurium-Typhi genomic chimera: a model to study Vi polysaccharide capsule function in vivo. Plos Pathog. 7 , e1002131 (2011).

37. Hollenbach, J.A. et al. Susceptibility to Crohn's disease is mediated by KIR2DL2/KIR2DL3 heterozygosity and the HLA-C ligand. Immunogenetics 61, 663-671 (2009). 